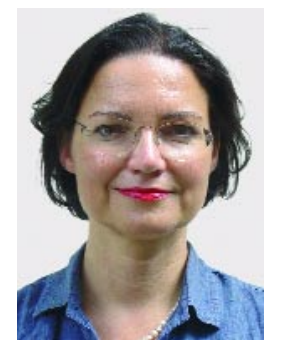

Gabriela Stoppe

\title{
Entlastung der Angehörigen von Demenzkranken durch ehrenamtliche Helfer
}

\author{
Gabriela Stoppe \& Petra Geilfuss
}

Bereich Allgemeine Psychiatrie, Psychiatrische Universitätsklinik Basel Gedächtnissprechstunde der Klinik und Poliklinik für Psychiatrie und Psychotherapie, Bereich Humanmedizin der Georg-August-Universität Göttingen

psychoneuro 2004; 30 (9): 505-508

$\mathrm{E}$ hrenamtliche Helfer (Laienhelfer) können die ambulante Versorgung von Demenzkranken sinnvoll verbessern und unterstützen. Der folgende Beitrag möchte das Konzept vorstellen und sich zum einen die Frage stellen, warum überhaupt Laienhilfe überlegt werden sollte, zum anderen bisherige Beispiele in der deutschsprachigen Versorgung nennen und kritische Punkte an der Darstellung des eigenen Projekts in Göttingen zusammenfassen.

\section{Warum überhaupt Laien- \\ hilfe?}

Epidemiologische Grundlagen

Die Epidemiologie demenzieller Erkrankungen stellt sich in verschiedenen Studien in verschiedenen Ländern sehr ähnlich dar und unterstreicht die Validität der Ergebnisse dieser Studien. Danach sind in Deutschland derzeit etwa eine Million Menschen betroffen. Nachdem sowohl die Krankheitshäufigkeit als auch die Neuerkrankungsrate mit zunehmendem Lebensalter exponentiell ansteigen, was im wesentlichen auf die Hauptursache Alzheimer Demenz zurückgeht, wird sich die Anzahl Erkrankter in den nächsten 30 Jahren verdoppeln. Dies hat mit dem zu erwartenden Anstieg der hochaltrigen Bevölkerung zu tun $(1,13)$.

\section{Demenz und Pflege}

Nachdem die Demenz oft auch die Ursache ist, dass ein alter Mensch für die körperlichen Erkrankungen

Die Zunahme von Demenzkranken in den nächsten Jahren wird begleitet von einer Verringerung der familiären Hilfemöglichkeiten. Die Familien tragen derzeit noch die Hauptlast der ambulanten Versorgung. Pflegende Angehörige haben ein hohes eigenes Erkrankungsrisiko. Professionelle Hilfe wird oft nicht in Anspruch genommen, weil sie nicht flexibel und niedrig schwellig genug ist, zudem teuer ist. Der Beitrag prüft, ob und wie intra- und intergenerationelle Hilfe durch Ehrenamtliche hier einen Beitrag leisten kann. Die bisherigen Beispiele zeigen, dass besonders eine zeitliche Entlastung von der Betreuungsaufgabe als hilfreich wahrgenommen wird. Laienhelfer sollten qualifiziert, versichert und entschädigt werden. Eine Identitätsbildung u.a. über Zertifikate ist hilfreich, auch in der Abgrenzung zur professionellen Hilfe, die sie nicht ersetzen kann und soll.

fremde Hilfe benötigt, ist sie häufig die (Haupt-)Ursache für Pflegebedürftigkeit. Schon heute sind etwa ein Drittel der Bewohner von Altenheimen und mehr als zwei Drittel der Bewohner von Pflegeheimen dement (14). Laut Rechenschaftsbericht 2003 des Verbandes der privaten Krankenversicherung e.V. (www. pkv.de) begründen Demenzen in der privaten Pflegepflichtversicherung den Pflegebedarf in Pflegestufe I bei $17 \%$, in Pflegestufe II bei $23 \%$, in Pflegestufe III und bei den Härtefällen jeweils bei $36 \%$. Bei den gesetzlichen Krankenkassen werden Demenzen als pflegebegründende Erstdiagnosen in $14,4 \%$ (Pflegestufe I), 16,9\% (Pflegestufe II) und 13,2\% (Pflegestufe III) genannt (persönliche Mitteilung des Medizinischen Dienstes der Spitzenverbände der Krankenkassen). Nachdem die stationäre Pflege teuer ist, sie zudem auch von den Angehörigen und den Patienten selbst oft als ungeliebte Zukunftsperspektive gesehen wird, ist die Heimeinweisung ein Ziel, dass es zu verhindern gilt. Dabei muss berück- sichtigt werden, dass sie in vielen Fällen auch eine Entlastung für alle Betroffenen bedeutet. Letztendlich wird heute die Endphase der Erkrankung in mehr als der Hälfte der Fälle in einem Heim verbracht (14).

\section{Die Einweisung in ein Heim}

Für die Heimweinweisung wurden in verschiedenen Untersuchungen Risikofaktoren identifiziert. Auf Seiten des Patienten waren ein höheres Lebensalter, Verhaltensstörungen und vor allen Dingen das „allein leben“ relevant, nicht so sehr die Demenzschwere. Auf Seiten der Pflegepersonen spielte insbesondere das Alter der Pflegeperson eine Rolle. Auch hier ist Hochaltrigkeit bedeutsam. Einen starken Risikofaktor bildet zudem die Belastung durch die Pflege, insbesondere Inkontinenz, Aggressionen und nächtliche Unruhe (15). Nach einer deutschen Untersuchung müssen 51\% der Pflegepersonen von Demenzerkrankten den Nachtschlaf regelmäßig unterbrechen, im Durchschnitt 2,4-mal pro Nacht (5). 


\section{Tab. 1 Aufgaben bzw. Tätigkeiten von Laienhelfern (Beispiele)}

- Gespräche mit Angehörigen

- Spaziergänge, Gespräche mit den Erkrankten

- Betreuung der Demenzkranken in deren Wohnung

- Spiele spielen

- Begleitung zu Arztterminen, zur Physiotherapie, bei Cafébesuchen, Friedhofsgängen, Kirchgängen etc.

- Kleinere Ausflüge

- Falls erforderlich Hilfestellung beim Anziehen, Toilettengängen, Nahrungsaufnahme

- Vermittlung von Gedächtnisstrategien wie Führen eines Terminkalenders

- Systematische Beschäftigung mit biographischem Wissen zur Erhaltung selbstbezogenen Wissens und aktuell bedeutender Erinnerungen

Die Gesundheit der Angehörigen

Es verwundert damit nicht, dass sich die Angehörigen bzw. Pflegepersonen durch die Betreuung bzw. Pflege mehrheitlich stark oder sehr stark belastet fühlen. Zwischen 30 und 50\% der betreuenden Angehörigen erkranken selbst körperlich und/oder seelisch, auch ist die Mortalität erhöht $(9,12)$. In einer Untersuchung im Rahmen des u.g. PRODEM-Projektes gaben nur zwei Prozent der Angehörigen an, sich wenig belastet zu fühlen und weitere 37\% gaben eine mäßige Belastung an (8). Die Angehörigen leiden dabei am meisten unter Zeitmangel und Verlust der Unabhängigkeit. Sie fühlen sich sozial isoliert und leiden unter dem Verlust bzw. der Veränderung der Beziehung („Ich kann meine Gedanken nicht mehr mit Kranken teilen“). Entsprechend wünschten sich in einer großen Untersuchung in Deutschland mit 69\% die meisten eine ideelle und materielle Anerkennung ihrer Pflegetätigkeit, gefolgt von nicht professioneller praktischer Hilfe. Medizinische Information und Beratung oder auch strukturelle Hilfe wünschten sich etwa ein Drittel der Befragten (4).

\section{Interventionen zur Entlastung der Angehörigen}

Bisher liegen nur wenige Interventionsstudien vor. Diese konnten belegen, dass Entlastungsangebote für die Angehörigen von Demenz- kranken wirksam sind (z.B. 3, 10, 11). So konnte gezeigt werden, dass eine individuelle und Familienberatung, zugeschnitten auf die spezifischen Probleme sowie die Teilnahme an einer wöchentlichen Gruppe und die jederzeitige Erreichbarkeit von Beratern im Vergleich zu einer Kontrollgruppe, die Beratung und Unterstützung nur auf Anfrage erhielt, das Risiko für eine Heimaufnahme in der Interventionsgruppe auf zwei Drittel des Risikos der Kontrollgruppe reduzierten (10). Vergleichende Untersuchungen verschiedener Interventionen zeigten, dass den Stress der Angehörigen vor allen Dingen eine Entlastung für wenige Stunden in der Woche verringerte (11).

\section{Ökonomische Daten}

Angehörige spielen eine große Rolle in der Versorgung von Demenzkranken. Nach deutschen gesundheitsökonomischen Berechnungen tragen die Familien derzeit knapp 70\% der gewichteten Gesamtkosten für einen Demenzpatienten. Auf die Krankenversicherer entfallen etwa $3 \%$, die übrigen Leistungen werden von der Pflegeversicherung erbracht (6). Man kann also leicht schätzen, welche Auswirkungen es hat, wenn die Familien nicht im derzeitigen Maße zur Verfügung stehen. Dies gilt vor allem für die Frauen, die mehrheitlich als Ehefrau, Töchter, Schwiegertöchter der Patienten diese Aufgabe übernehmen. Prospektiv muss die epidemiologische Entwicklung ebenso berücksichtigt werden, wie die der Sozialstrukturen. Es zeigt sich jetzt schon, dass in den nächsten Jahren die Anzahl der allein Lebenden zunehmen wird. Dies zeigt sich jetzt schon im (groß-) städtischen Bereich. Im Vergleich zu heute werden ältere Männer in der Zukunft häufiger allein leben, Frauen eher weniger. Die geringere Anzahl von Kindern, die größere Rate an Berufstätigkeit und die Verringerung des räumlichen $\mathrm{Zu}$ sammenlebens von Familien lassen zumindest eine „selbstverständliche“ Pflege aus dem Familienkreis zunehmend unrealistisch werden. In der Gesellschaft kommen heute auf einen Demenzkranken 69,4 Personen im Erwerbsalter. Im Jahr 2050 werden es nur 21,1 sein. Daran würde auch ein sofortiger deutlicher Anstieg der Fertilität nichts Wesentliches ändern (13).

Es stellt sich somit die Frage, ob eine Entlastung bzw. Unterstützung des Betreuungsaufwandes von Demenzkranken durch eine Förderung der inter- und intragenerationellen Hilfe, unter anderem auch durch Ehrenamtliche, geleistet werden kann.

\section{Wie sieht Laienhilfe aus?}

Laienhelfer, dies sagt schon der Name, sind keine Professionellen. Sie dürfen somit mit keinerlei Aufgaben betraut werden, die eine professionelle Kompetenz im Pflegebereich erfordern. Kritisch wird die Situation in der Tat, wenn z.B. teilstationäre oder stationäre Pflegeeinrichtungen durch die Beschäftigung bzw. Vermittlung von Laienhelfern unter Umständen ihr eigenes Pflegepersonal von nicht zwingend pflegerischen Betreuungsaufgaben entlasten wollen. Auch die Abgrenzung zu anderen Professionellen in diesem Bereich, insbesondere Ergo- und Physiotherapeuten, muss sorgfältig erfolgen. Das Spektrum der Tätigkeit von Laienhelfern sollte immer in Absprache mit den entsprechenden Familien bzw. Betreuern erfolgen und auch den Wünschen und Ansprüchen der Ehrenamtlichen entgegenkommen. So gehören hierzu Spaziergänge oder Vorlesen etc. dazu (Tab. 1). Betreuungsgruppen, Helferinnenkreise, auch Urlaubsangebote finden sich ebenfalls im Bereich der ambulanten Versorgungsformen unter Beteiligung von Laien (7).

Laienhelfer sollten Kenntnisse von dem Krankheitsbild der Demenz haben und auch wissen, wie man mit schwierigen Situationen umgeht (2). Was soll z.B. passieren, wenn der Demenzkranke sich weigert, über die Straße zu gehen oder wenn der Demenzkranke plötzlich den Laienhelfer verkennt und nicht mehr akzeptiert? Dies verlangt eine Schulung, die jedoch nicht gleich zur vollen Professionalisierung beiträgt. Inhalte der Schulung können und sollten sein:

- Situation pflegender Angehöriger

- Krankheitsbild der Demenz

- Umgang mit Verwirrtheit, Aggressivität etc.

- Gesprächsführung

- Sozialrechtliche Fragen 
- Einführung in die Aktivitäten des täglichen Lebens.

Im Folgenden sollen Beispiele aus dem deutschsprachigen Raum einen Einblick in das Spektrum von Laienhilfe geben.

Die Angebote der Memoryklinik Entlisberg in Zürich: Diese Einrichtung hat eine Reihe von Angeboten für die Entlastung von Angehörigen von Demenzkranken entwickelt. Hierzu gehören z.B. regelmäßig stattfindende Tanzcafes, zu denen Angehörige mit ihren Demenzkranken kommen können. Zudem werden sogenannte Spaziertherapeuten eingesetzt. Diese organisieren nach einer vorherigen Schulung entweder individuell oder in Gruppen regelmäßig Spaziergänge mit Demenzkranken. Durch ein Zertifikat und auch durch regelmäßige Treffen ist ein Gruppengefühl unter den Spaziertherapeuten entstanden.

„Für ein paar Stunden Urlaub“ der Angehörigenberatung e.V. in Nürnberg: Seit Mitte der 90-er Jahre wurden durch die Angehörigenberatung e.V. Nürnberg, später auch in anderen Regionen Bayerns und Baden-Württembergs Helfer ausgebildet. In einem 40-stündigen Curriculum werden sie mit der Aufgabe vertraut gemacht, erhalten ein Zertifikat und treffen sich monatlich. Einmal jährlich wird eine Fortbildung angeboten. Die Aufwandsentschädigung beträgt 7,70 € pro Stunde, die Versicherung der Helfer trägt der ausbildende Verein. Die Vermittlung der Laienhelfer erfolgt über die Beratungsstelle. Auch hier ist die Entwicklung einer gemeinsamen Identität der Helfer wichtig.

Das Projekt PRODEM in Stuhr/Weyhe: Dieses Projekt wurde durch die Firma Schwabe gefördert und durch ein unabhängiges Institut evaluiert. An dem Projekt nahmen zehn Praxen teil, die vorher eine spezielle Informationsveranstaltung zur Demenz besucht hatten. Die Ärzte wurden von delegierbaren Verwaltungs- und Koordinationsaufgaben durch speziell eingestellte Projektkoordinatoren entlastet. Diese organi- sierten Hausbesuche, in denen der Betreuungsbedarf festgestellt wurde. Auch fanden regelmäßig „,runde Tische“ der an der Versorgung Beteiligten statt. Ab 1999 wurden „Gesellschafter", die an vier Terminen fortgebildet wurden, für die ehrenamtliche Unterstützung gewonnen. Nach Ablauf der Förderung wurde das Projekt nach unserer Kenntnis nicht weiter fortgeführt.

Das Projekt „Lichtblick“ des Alexianer Krankenhauses in Münster: Diese Initiative wurde drei Jahre durch das Bundesministerium für Gesundheit unterstützt. „Freiwillige Helfer" wurden in einem 25(später 30-)stündigen Curriculum für die Aufgabe in den „häuslichen Besuchsdiensten" ausgebildet. Auch sie erhalten ein Zertifikat und anschließende Möglichkeiten zu Treffen und Supervision. Das Projekt ist mit dem gerontopsychiatrischen Zentrum verbunden. Eine Entschädigung der Laienhelfer war zunächst nicht vorgesehen, konnte dann aber ermöglicht werden. Das Angebot ist trägerübergreifend.

Nicht genauer sollen und können hier weitere Projekte beschrieben werden, die auf lokale Initiativen hin durch gemeinsame Aktionen verschiedener Institutionen (z.B. Gesundheitsamt, Beratungsstellen, Krankenkassen, Krankenhäuser, Arztpraxen ...) auf die Nöte der Demenzkranken und ihrer Angehörigen hinweisen und Hilfen ermöglichen, wie dies in Stade oder Braunschweig und anderen Orten der Fall ist.

\section{Schlussfolgerungen aus den geschilderten Projekten und Darstellung des Göttinger Projektes zur Laienhilfe}

Mehrere Faktoren sind für den Erfolg einer Laienhilfe bedeutsam. An erster Stelle scheint hier die erfolgreiche Rekrutierung zu stehen. Mindestens genauso wichtig ist es jedoch auch, die angeworbenen Personen langfristig zu motivieren. Hierbei scheint zum einen eine sichtbar vermittelte Qualifikation (Zertifikat), zum anderen eine Förderung der Laienhelfer-Identität (durch Verein, Gruppenbildung, Supervision, regelmäßigen Erfahrungsaustausch) eine wichtige Rolle zu spielen. Zum Zweiten ist es wichtig, dass die versicherungsrechtliche Seite geklärt ist. Dies scheint dann gewährleistet, wenn z.B. ein Verein oder ein anderer Träger die Versicherung übernimmt. Drittens ist es bedeutsam, dass die Abgrenzung zur professionellen Unterstützung klar getroffen wird. Wie dies geschieht, wird nicht immer klar ausgeführt, dennoch ist die Klarheit über Aufgaben und Pflichten sowohl auf Seiten der in Anspruch nehmenden Angehörigen als auch der Laienhelfer von Relevanz. Viertens ist das Ineinandergreifen der Versorgung bedeutsam, was konkret bedeutet, dass die Laienhilfe in der Regel die Schwelle zur Inanspruchnahme durch die Betroffenen senkt und dadurch erlaubt, schon früh - gegebenenfalls auch professionelle Unterstützung - zu vermitteln und wirksam werden zu lassen. Fünftens ist eine Verankerung in der lokalen „Szene“ unabdingbar.

In Göttingen haben wir im Jahr 2001 eine entsprechende Kooperation etabliert. Die Gedächtnissprechstunde der Universität, die seit über zehn Jahren erfolgreich in der Frühdiagnostik und frühen Beratung von Demenzkranken tätig ist, trat in Kooperation mit dem Diakonieverband in Göttingen. Somit ist nach unserer Kenntnis erstmals eine medizinische Einrichtung, die bereits in der frühen Krankheitsphase Hilfe vermitteln will, entscheidend eingebunden. Das Projekt wird zudem unterstützt durch die Stadt, die Alzheimer Gesellschaft und - z.B. zur Förderung der Öffentlichkeit - durch die Apotheken, die niedergelassenen Ärzte und die Presse. Die Finanzierung für die Begleitforschung und die Schulung er-

\section{Tab. 2 Noch offene Fragen in der Laien-} hilfe für Demenzkranke

- Was sichert die Nachhaltigkeit der Maßnahmen, insbesondere welche Laienhelfer bleiben „dabei”?

- Welches Ausbildungs-Curriculum ist optimal? Sind Nachschulungen sinnvoll?

- Gibt es Demenzkranke bzw. Angehörige, die durch dieses Angebot nicht erreicht werden können? Wodurch sind sie gekennzeichnet?

- Wie wirkt sich die Laienhilfe auf die Gesundheit der Angehörigen, der Demenzkranken und auch der jeweiligen Laienhelfer aus? 


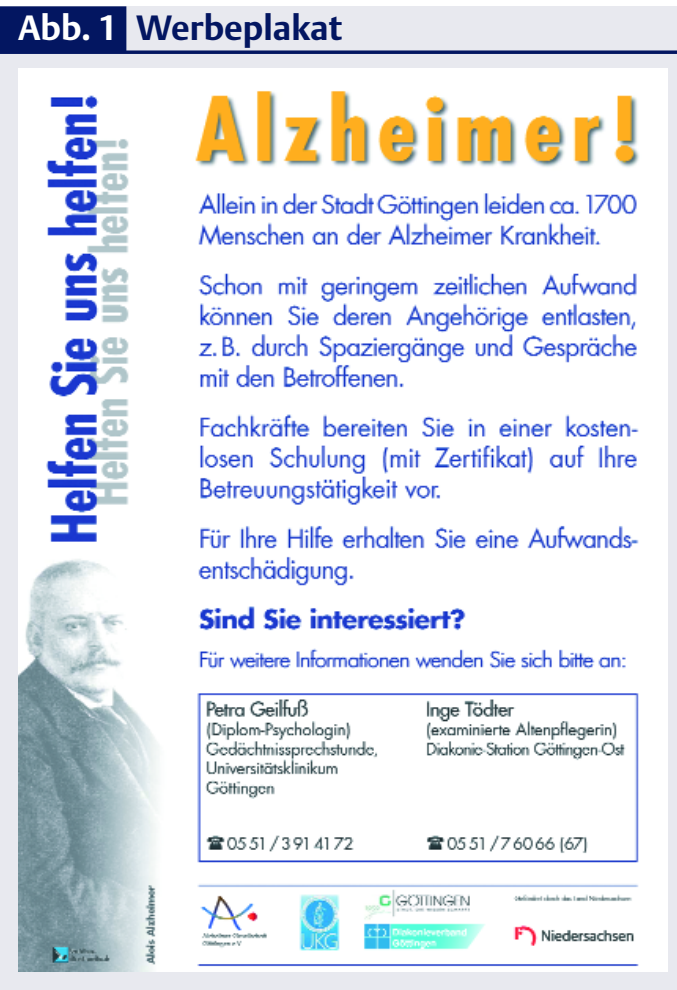

Plakat für die Anwerbung von Laienhelfern, wie es in Göttingen zum Einsatz kam.

folgt durch das niedersächsische Ministerium für Frauen, Arbeit und Soziales. Die öffentliche Resonanz auf ein Plakat (s. Abb. 1) und Presseartikel war groß. Laien, die sich für den Einsatz melden, werden nach einem Eingangsinterview und Beratungsgesprächen einer 40-Stunden-Schulung zugeführt. Hierfür wird ein Zertifikat ausgestellt. Nur Personen die dieses Zertifikat erworben haben, werden durch den Diakonieverband für die ambulante Betreuung vermittelt. Die Vermittlung eines Laienhelfers erfolgt auch erst dann, wenn eine professionelle Pflegekraft sich bei einem Hausbesuch vergewissert hat, dass hier nicht professionelle Pflege durch Laienhilfe ersetzt werden soll. Die Versicherung der Laienhelfer erfolgt ebenfalls über den Diakonieverband. Die Entschädigung in Höhe des ehrenamtlichen „Honorars“ (7,50 €) erfolgt direkt durch die in Anspruch nehmenden Angehörigen. Hier werden inzwischen auch Mittel, die nach dem Pflegeleistungsergänzungsgesetz zur Verfügung gestellt werden können, angewendet. In einer Begleitforschung untersuchen die Autorinnen im Längsschnitt, welchen Einfluss diese Maßnahme auf die Gesundheit der Demenzkranken, der Angehörigen und auch der Laienhelfer hat. Diese und weitere Forschungsthemen finden sich in Tabelle 2.

\section{Abschließende Bemerkungen}

Laienhilfe scheint unter bestimmten Bedingungen eine erfolgreiche und alle Seiten zufriedenstellende Möglichkeit zu sein, die ambulante Versorgung von Demenzkranken zu verbessern und auch zu unterstützen. Sie ersetzt nicht professionelle Hilfe. Ihr besonderer Vorteil ist die hohe Flexibilität. Dadurch wird die Schwelle zur Inanspruchnahme gesenkt, was wiederum dazu führt, dass Betroffene früher Kenntnis von Hilfsmitteln erhalten. So könnte Laienhilfe auch dazu beitragen, krisenhafte Situationen in der ambulanten Versorgung zu verhindern.

\section{Relief of dementia caregiving by layhelp}

The increasing numbers of patients suffering from dementia within the next decades will be accompanied by delegate rates of family ressources for informal care. However, family care is the most relevant form of ambulatory care. Caregivers have an increased risk of own morbidity and mortality. They often do not ask for professional help, because they recognise it as to rigid, invasive and costly. The article focuses on whether inter- and intragenerational lay help may be useful in this situation. Available evidence shows that the opportunity to declining care responsibilities for several hours is especially welcome. Lay helpers should be qualified, insured and paid. A „corporate identity " should be builded which also helps with regard to separation from professional care and help, which cannot and should not be replaced.

\section{Key Words:}

dementia - lay help - ambulatory care - caregiving

\section{Literatur}

1. Bickel H. Demenzsyndrom und Alzheimer Krankheit. Eine Schätzung des Krankenbestandes und der jährlichen Neuerkrankungen in Deutschland. Gesundh Wes 2000; 62: 211-218

2. Franke L, Kämmer K. Pflege und Betreuung Demenzkranker. In: Wächtler C (Hrsg.).
Demenzen. Stuttgart: Georg Thieme, 2003: 72-83

3. Gaugler JE et al. Adult day service use and reductions in caregiving hours: effects on stress and psychological well-being for dementia caregivers. Int J Geriat Psychiatry 2003; 18: 55-62

4. Gräßel E. Belastung und gesundheitliche Situation der Pflegenden. Querschnittsuntersuchungen zur häuslichen Pflege bei chronischem Hilfs- und Pflegebedarf im Alter. Egelsbach: Hänsel-Hohenhausen (Deutsche Hochschulschriften; 1134), 1997

5. Gräßel E. Warum pflegen Angehörige? Ein Pflegemodell für die häusliche Pflege im höheren Lebensalter. Zschr Gerontopsychol \& psychiatr 2000; 13: 85-94

6. Hallauer JF, Schons M, Smala A, Berger $\mathrm{K}$. Untersuchungen von Krankheitskosten bei Patienten mit Alzheimer-Erkrankung in Deutschland. Gesundh okon Qual manag 2000; 5: 73-79

7. Jansen S. Ambulante Versorgungsformen. In: Hallauer JF, Kurz A (Hrsg). Weissbuch Demenz. Stuttgart: Georg Thieme, 2002: 74-77

8. Klingenberg A, Szecsenyi J. Unterstützungsbedarf von pflegenden Angehörigen. Befragungsergebnisse von Familien Demenzkranker in einer ländlichen Region bei Bremen. Z Alllg Med 1999; 75: 1113-1118 9. Langa KM et al. National Estimates of the Quantity and Cost of Informal Caregiving for the Elderly with Dementia. J Gen Intern Med 2001; 16: 770-778

10. Mittelman MS et al. A family intervention to delay nursing home placement of patients with Alzheimer disease. A randomized controlled trial. J Am Med Assoc 1996; 276: 1725-1731

11. Quayhagen MP et al. Coping with dementia: evaluation of four nonpharmacological interventions. Int Psychogeriatr 2000; 12: 249-265

12. Rainer $\mathrm{M}$ et al. Pflegende Angehörige von Demenzerkrankten: Belastungsfaktoren und deren Auswirkung. Psychiat Prax 2002; 29: 142-147

13. Wancata J et al. Number of dementia sufferers in Europe between the years 2000 and 2050. Eur Psychiatry 2003; 18: 306-313 14. Weyerer $S$ et al. Demenzkranke in Einrichtungen der voll- und teilstationären Altenhilfe. Epidemiologische Forschungsergebnisse. In: Sozialministerium BadenWürttemberg (Hrsg): Weiterentwicklung der Versorgungskonzepte für Demenzerkrankte in (teil-)stationären Altenhilfeeinrichtungen. Stuttgart, 2000: 1-58

15. Yaffe $\mathrm{K}$ et al. Patient and caregiver characteristics and nursing home placement in patients with dementia. J Am Med Ass 2002; 287: 2090-2097

\section{Korrrespondenzadresse:}

Prof. Dr. med. Gabriela Stoppe

Ärztliche Leiterin des

Bereichs Allgemeine Psychiatrie

Psychiatrische Universitätsklinik Basel

Wilhelm Klein-Strasse 27

CH-4025 Basel

E-Mail: gabriela.stoppe@pukbasel.ch 